\title{
Schweizerische Theophrastforschung und schweizerische Theophrasteditionen im I6. Jahrhundert und ihre Bedeutung
}

I.

Im Altertum gab es eine botanische Wissenschaft, die mit Theophrastos von Eresos (370-285 v. Ch.) ihren Höhepunkt erreicht hatte. Als im Verlauf des mithridatischen Kriegs Athen fiel und Sulla die Handschriften Theophrasts als kostbare Beute nach Rom überführen ließ, wurden diese durch viele Abschriften in Italien offenbar ziemlich rasch in weitern Kreisen bekannt. Laertius, ein Biograph des großen griechischen Gelehrten, will noch im 3. Jahrhundert gegen zweihundert verschiedene Theophrastschriften gekannt haben. ${ }^{1}$ In den Wirren der Völkerwanderung sind fast alle zugrunde gegangen; nur ein geringer Bruchteil hat sich bis auf unsere Tage erhalten. Auch diese sind dem Mittelalter unbekannt gewesen. Sie kamen erst im ausgehenden 15. und im 16. Jahrhundert wieder ans Tageslicht und damit bald auch in den Druck.

Das Mittelalter hat es zu keiner wissenschaftlichen Botanik gebracht, nur zu einer praktisch orientierten Pflanzenkunde. Diese wurde zuerst in Klöstern gepflegt; Reichenau und St. Gallen waren vom 8.-11. Jahrhundert bedeutende Kultur- und Bildungszentren. Nach der Gründung eigentlicher Medizinschulen, vor allem der Schulen von Salerno und Montpellier, wurde die Pfanzenkunde mehr und mehr ein Teilgebiet der Medizin. ${ }^{2}$ Da die Schweiz im spätern Mittelalter kein solches Bildungszentrum besaß - die Basler Hochschule wurde erst um die Mitte des 15. Jahrhunderts gegründet - hat sie in dieser Epoche keinen Beitrag zur Pflanzenkunde geleistet.

Im 16. Jahrhundert finden sich im Abendland die ersten Ansätze zur Ausbildung der modernen botanischen Wissenschaft. Sie hat den abendländischen Rahmen in der Folge gesprengt und sich über alle Kulturkreise der Erde ausgebreitet. Wo überall ihre Betrachtungsweise und Denkmethode angenommen wurden, faßte sie festen Fuß. An sich wären natürlich auch anders geartete denkbar gewesen; aber nur diese haben zur Ausbildung einer Wissenschaft geführt. Die noch heute gültige Betrachtungsweise und Denkmethode - erneut - zur Anerkennung gebracht zu haben, ist das Verdienst der Gelehrten des 16. Jahrhunderts. An dieser Entwicklung hatten zunächst die deutsche Schweiz mit dem angrenzenden alemannischen Elsaß und Frankreich, nachher vor allem 
die Niederlande und Italien führenden Anteil. Sie setzte ein mit dem Bekanntwerden der biologischen Schriften Theophrasts. In der gleichen Zeit, in der die oben genannten Länder in besonderm Maß zum Ausbau der jungen Wissenschaft beitrugen, leisteten sie auch ihren entscheidendsten Anteil an der zeitgenössischen Theophrastforschung, wobei zwischen den einzelnen Kulturkreisen enge Beziehungen bestanden und manche $\mathrm{Ge}$ lehrte dem einen wie dem andern angehörten. ${ }^{3}$

Bevor eine solche Entwicklung einsetzen konnte, welche die mittelalterliche Pflanzenkunde in eine botanische Wissenschaft überführte, mußten verschiedene Bedingungen erfüllt sein, eine Leistung, welche das 15. Jahrhundert vollbrachte. Diese soll zunächst in Kürze geschildert werden.

\section{II.}

Die Erfindung von Buchdruck und Holzschnitt waren der Verbreitung pflanzenkundlicher Kenntnisse natürlich sehr förderlich. Schon im 15. Jahrhundert kamen illustrierte Kräuterbücher in verschiedenen Sprachen auf den Markt. Auch die mittelalterlichen Enzyklopädien eines Bartholomaeus Anglicus und Thomas Cantipratensis wurden frühzeitig gedruckt und dienten ihrerseits der Verbreitung naturkundlichen Wissens. Nicht nur Klöster und Stifte, auch Einzelpersonen schafften sich solche Werke an; von des Bartholomaeus Büchern «de proprietatibus rerum» besitzt die Zürcher Zentralbibliothek nicht weniger als sechs Inkunabeln, von denen eine z. B. zuerst einem Schulmeister von Beromünster, nachher einem Klerikerarzt gehört hatte. ${ }^{4}$

Von nicht geringerer Bedeutung war ein anderes Moment, das die Entwicklung der beschreibenden Naturkunde, vorab der Botanik, besonders begünstigte: ein wirklichkeitsnäheres Sehen, das sich in einer objektgetreueren Darstellung äußerte. Noch bis ins 16. Jahrhundert findet man Pflanzenabbildungen, die ohne große Ähnlichkeit mit dem abzubildenden Gegenstand in einer bestimmten Manier, nach festgelegtem Schema, hergestellt wurden. Sollte z. B. die Wurzel einer Pflanze abgebildet werden, hatte der Zeichner meist gar nicht nötig, sich dieselbe erst näher anzusehen, wußte er doch ohnehin, wie eine Wurzel dargestellt wird, nicht anders als etwa heute jedes Kind weiß, daß die Sonne als Kreis mit radiären Strahlen gezeichnet wird. Es handelte sich also weit weniger um Stilisierung als um Manier. Die Pflanzenbilder des Mittelalters waren zudem weit mehr symbolisch 
als impressionistisch gedacht. Die Ähnlichkeit eines Bildes mit dem dargestellten Gegenstand war im Grunde genommen weniger wichtig als etwa die Tatsache, daß im Bild die medizinische Verwendung oder etymologische Beziehungen zum Ausdruck kamen. So wurde Conrad Geßner von einem Korrespondenten ein Bild des Skorpionskrautes (Coronilla scorpioides) geschickt, welches wegen angeblicher Ähnlichkeit der Hülsen mit Skorpionen gegen Skorpionsbiß verwendet wurde, auf dem auch die Wurzeln als ein Netz von Skorpionen dargestellt waren; das Bild hat sich in seinem botanischen Nachlaß erhalten. ${ }^{5}$ Ein wirklichkeitsnäheres Sehen machte sich zuerst in der Kunst geltend. Es findet sich anfänglich in der italienischen und flämischen, um die Jahrhundertwende aber auch bereits in der deutschen Kunst; Jan van Eyck, Lionardo da Vinci und Albrecht Dürer sind dafür gültige Zeugen, doch zeigt es sich auch in unzähligen Miniaturen in flämischen und italienischen Stundenbüchern. Führte ein wirklichkeitsnäheres Sehen zu neuer Art der Darstellung, erzog diese ihrerseits wiederum das Sehen. Denn der mittelalterliche Mensch mußte erst zu diesem Sehen erzogen werden. Nicht nur die Pflanzenkunde, auch die Tierkunde, die Anatomie, ja jede beschreibende Naturwissenschaft wurde von diesem objektgetreueren Sehen gefördert und verdankte ihm im 16. Jahrhundert weitgehend den großartigen Aufschwung. Mit vollem Recht bemerkt $H$. Fischer in seiner mittelalterlichen Pflanzenkunde, die realistische Pflanzendarstellung habe sich in einem Jahrhundert zu einer Höhe entwickelt, daß sie auch später nicht mehr überboten werden konnte. ${ }^{6}$

Neben diesem realistischeren Sehen kündigte sich im 15. Jahrhundert - zunächst wieder in Italien - eine neue, dem Mittelalter durchaus fremde geistige Haltung an, besonders bei Gelehrten, die sich mit Realwissenschaften beschäftigten. Mit der Formel, es habe sich für jene Gelehrten darum gehandelt, die Wissenschaft der Alten in ihrer ursprünglichen Form, befreit von allen arabischen Zusätzen, wieder herzustellen, weil sie angeblich des kindlichen Glaubens waren, die Alten hätten die ganze Wissenschaft schon besessen und es handle sich nur darum, wieder in ihren Besitz zu kommen, wird man sich einem Verständnis jener geistigen Situation sicher nicht nähern. Seit Petrarca pflegten die italienischen Humanisten das Ideal einer «Plenitudo temporum» als Bildungsziel: antike Weisheit, durchleuchtet von christlichem Geist, in schönem, römisch-ciceronischem Gewand. Dieses Ideal der Weisheit war im Abwehrkampf gegen das glaubensbedrohende rationale Ideal averroistischer 
Wissenschaft, das das platonische Erbe bedrohte, entstanden. In der Folge gewann das national-römische Kulturprinzip immer mehr die Oberhand; vornehmlich durch philologische Arbeit wurde nun versucht, ihm neben der antiken Weisheit auch die antike Wissenschaft einzugliedern. Dieses überspitzte nationalistisch-römische Kulturprinzip blieb nicht ohne Folgen, auf dem Gebiet der Religion wie der Wissenschaft. Dem römischen wurden bald andere nationale Kulturprinzipien entgegengestellt. So waren die griechischen Gelehrten in Italien, die um die Jahrhundertmitte nach der Einnahme von Konstantinopel durch die Türken in großer Zahl nach Italien emigriert waren, immer weniger Willens, das große griechische Erbe in den Dienst eines italienisch-römischen Kulturprimats zu stellen; sie stellten ihm ihr griechisches gegenüber. Der Kreis um Kardinal Bessarion und der aldinische Verlag waren die Brennpunkte dieser Bestrebungen. ${ }^{7}$

Im Zeichen dieses auf italienischem Boden sich abspielenden Kulturkampfes wurden dem Abendland die biologischen Werke Theophrasts neu geschenkt. Ein griechischer Arzt und Humanist, Theodor Gaza, der noch im 14. Jahrhundert zur Welt gekommen und nach der Einnahme seiner thessalonischen Heimat nach Italien ausgewandert war, hatte von Papst Nicolaus V. den Auftrag bekommen, dieselben in lateinischer Sprache zu edieren, eine Aufgabe, die viele Schwierigkeiten machte, einmal, weil nur eine zwar ziemlich vollständige, aber reichlich verderbte Handschrift zur Verfügung stand, dann aber auch, weil viele der von Theophrast erwähnten, aber ungenügend beschriebenen Pflanzen nur in Griechenland, nicht aber in Italien vorkamen und keine lateinischen Namen besaßen. Der Umstand, daß Gaza ohne Zweifel selber keine wesentlichen pflanzenkundlichen Kenntnisse besaß, erschwerte seine Arbeit natürlich. ${ }^{8}$ Plinius, seine einzige Stütze, ließ ihn vielfach im Stich. Auch er hatte sich seinerzeit derselben Schwierigkeit gegenüber gesehen und so geholfen, daß er die griechischen Pflanzennamen einfach durch ähnlich lautende lateinische Worte wiedergab, wodurch der Sachverhalt natürlich nicht aufgehellt wurde. ${ }^{9}$ In den achtziger Jahren - Gaza war damals bereits tot - erschienen diese Werke in ziemlich unvollkommener Form. ${ }^{10}$ Im folgenden Jahrzehnt bemächtigte sich der aldinische Verlag derselben und gab sie, ebenfalls in der Interpretation von Gaza, in griechischer Sprache heraus und zwar in einer Prachtausgabe. ${ }^{11}$ Wenige Jahre später ließ er auch eine lateinische Ausgabe in der Übertragung Gaza's folgen; durch eine Kapiteleinteilung und ein Vokabular war diese 
Ausgabe den frühern weit überlegen. Die griechische Ausgabe blieb aber für die spätere Theophrastforschung grundlegend. Die römische Eingliederung war mißlungen. Doch bevor wir auf die Bedeutung dieser Publikation eingehen wollen, kehren wir zu unserm Ausgangspunkt zurück, zur geistigen Haltung der damaligen Gelehrten, die sich mit Pflanzenkunde beschäftigten.

$\mathrm{Da} ß$ die Philologie für das damalige römische Kultur- und Bildungsziel eine große Rolle spielte zur Assimilierung des antiken Schrifttums, wurde bereits erwähnt. So gab es natürlich auch rein philologische Bearbeiter der neu im Druck erscheinenden antiken Pflanzenwerke, wie etwa Hermolaus Barbarus (gest. 1493) ${ }^{12}$ und Marcellus Vergilius (gest. 1521). Die überlieferten Texte waren ja teilweise auch so korrumpiert, daß eine solche Tätigkeit nicht nur gerechtfertigt, sondern eine dringende Notwendigkeit war. Der Mangel an realen Sachkenntnissen erschwerte die Arbeit dieser Gelehrten und führte zu manchem Irrtum. Daneben gab es aber auch Gelehrte, die durchaus darüber verfügten und sich in kritisch positivistischem Sinn mit dem Inhalt solcher Werke beschäftigten, wobei ihr Interesse zunächst in erster Linie Plinius und Dioscurides galt; zu diesen gehörten Nicolaus Leonicenus (1428-1524), Medizinprofessor in Ferrara ${ }^{13}$ und Alexander Benedictus (gest. 1525), ${ }^{14}$ der um die Jahrhundertwende Pliniusausgaben besorgte. Leonicen stand den antiken Autoritäten vollkommen unbefangen gegenüber; er freute sich über jeden Schritt, den er über Plinius hinaus tun konnte, publizierte eine Schrift über zweihundert Irrtümer dieses Gelehrten und schrieb 1491 an seinen Freund Angelus Politianus, den bedeutenden Humanisten an der Florentiner Akademie: «Humanum est labi, decipi et errare; et tamen Plinius de hominum genere optime meritus». ${ }^{15}$

Dem Beispiel dieser geistigen Haltung folgten schon im beginnenden 16. Jahrhundert auch deutsche und schweizerische Humanisten, teilweise unter direktem Einfluß Leonicens. Voraussetzung für eine solche Entwicklung war eine rasch zunehmende Bildungsdichte dieser Länder im 15. Jahrhundert. Besonders gut bekannt sind die Verhältnisse in den Bistümern Chur ${ }^{16}$ und Konstanz, ${ }^{17}$ im letztern besonders diejenigen des Kantons St. Gallen. ${ }^{18}$ Von $1380-1419$ sind aus St. Gallen, das in der Ostschweiz die größte Bildungsdichte aufwies, 33 Hochschulstudenten bekannt, von 1420 - 1459 deren 119 , von 1460 - 1499 ihrer $328 .^{19}$ Die Gründung der Basler Hochschule hat zu diesem Aufschwung wesentlich beigetragen. Mit der zunehmenden Bildungsdichte, vor allem auch unter 
den Laien, wuchs auch das Kulturbewußtsein dieser Länder. Die Überheblichkeit vieler italienischer Humanisten an deutschen Hochschulen als Vertreter eines römisch-nationalen Kulturprimats wenn nicht gar Monopols, führte mehr und mehr zu einem ebenso bewußten deutschnationalistischen Kulturbewußtsein. Erasmus, der Fürst der germanischen Humanisten, anerkannte das italienische Kulturprimat nicht und ließ sich trotz verlockender Angebote demselben nicht dienstbar machen. ${ }^{20}$ Das griechische Kulturideal, wie auch das ursprüngliche Ideal einer Plenitudo temporum mit stark christlichem Einschlag, fand in ihm außerhalb Italiens den bedeutendsten Vertreter, während Konrad Celtis ein bewußt deutscher Humanist war, ein Romantiker, der sich als erster für das deutsche Kulturerbe interessierte. ${ }^{21}$ Von ihm wie von Leonicen beeinflußt wirkte an der Wiener Hochschule der St. Galler Humanist Vadian, der sich dort mehr und mehr zum Führer der deutschen Humanisten aufschwang, gleichzeitig aber völlig unbefangen mit den Werken antiker Autoritäten befaßte, ganz im Sinne Leonicens, freilich nicht mit botanischen, sondern mit geographischen. ${ }^{22}$ Er hatte Scholien zu Pomponius Mela verfaßt und zwar darob in Streit mit seinem ebenfalls an der Wiener Hochschule wirkenden Kollegen Camers geraten, der Italiener war. In seiner Verteidigungsschrift gegen Camers führte er aus, wenn er richtig sehe und ihn eine alte Erfahrung nicht täusche, habe er sich vor allem dadurch versündigt, daß er, ein bloßer Deutscher, es gewagt habe, anderer Meinung zu sein als Italiener, nämlich Plinius und Solinus. Daß sich Plinius in gewissen Dingen geirrt habe, spiele dabei ganz offensichtlich eine nebensächliche Rolle. Obwohl er selber keinen einzigen Schriftsteller häufiger und lieber lese als eben diesen, wisse er doch, daß auch er sich geirrt habe. Plinius selber habe ihn gelehrt, daß es Menschenlos sei, nicht allwissend und dem Irrtum unterworfen zu sein. So hoch er Plinius auch schätze: Homo fuit, errare et labi potuit. Er geißelt die Torheit, die Autorität über die Wahrheit stellen zu wollen und führt eine stattliche Reihe geographischer Irrtümer antiker Schriftsteller auf und meint, daß modernen Berichten von Augenzeugen weit mehr zu trauen sei als den Berichten der Alten, die lange nicht alles gewußt und sich oft geirrt hätten. Für diese Haltung beruft er sich mehrmals direkt auf Leonicen. ${ }^{23}$ Conrad Geßner, der nicht mehr als Humanist zu bezeichnen ist, wurde noch von R. Burckhardt in seiner Geschichte der Zoologie unter den philologischen Naturforschern aufgeführt, ${ }^{24}$ obwohl sich derselbe schon als fünfundzwanzig- 
jähriger Gelehrter gegen eine solche Unterstellung gewehrt hat durch ein wissenschaftliches Credo, das er mit Sätzen aus Galens Werken in einem Erstlingswerk nieder gelegt hat und von denen nur einige wenige angeführt werden sollen, weil sie seine eigene Einstellung besonders deutlich dokumentieren:

Sine contentiosis nominibus, ex rebus ipsis potius utilitatem consequi oportet.

De nominibus contendere caeteris permittimus; nobis enim res quas illi penitus ignorant, pertractare satis fuerit.

Meminisse oportet eius quod semper repetere solemus, ut quantum vocabula attinet pacem inter nos quamprimum statuamus atque ad res ipsas accelerentes in ipsis versemur diuque immoremur.

Nomina pro arbitrio mutare licebit, modo observes ut res ipsae permaneant. Etc. ${ }^{25}$

III.

Sowohl die Scholastik, diese bedeutende abendländische Schule rationalen Denkens, wie auch der Humanismus, die vielgestaltige, immer mehr national differenzierte Bildungsbewegung, die zu einem neuen Gelehrtentypus geführt hatte, haben das eine gemeinsam: ihre naturwissenschaftliche Beschäftigung - wenigstens was sie unter einer solchen verstanden - beschränkte sich auf eine Beschäftigung mit antiker naturwissenschaftlicher Literatur und führte nie zu einer solchen mit den Naturobjekten selbst. Albertus Magnus war eine große Einzelerscheinung, die in dieser Beziehung keine Folgen zeitigte. Wenn das 15. Jahrhundert für eine beschreibende Pflanzenkunde gleichwohl einige sehr bedeutende Ansätze zeigte, in erster Linie auf dem Gebiet der Kräuterbücher, welche die mittelalterlichen Erzeugnisse teilweise erheblich übertrafen, war das ein Verdienst des Buchdrucks mit seinen neuen Abbildungsmöglichkeiten; gerade in Abbildungen von einheimischen Pflanzen, z. B. in der Mainzer Erstausgabe des «gart der gesuntheit» zeitigte das naturgetreuere Sehen bereits sehr beachtenswerte Leistungen, gibt es doch nur sehr wenige Werke des Mittelalters, die mit wissenschaftlicher Absicht eine ähnlich naturgetreue Wiedergabe des Gesamthabitus einer Pflanze anstrebten und erreichten..$^{26}$ Man kann dabei aber nicht übersehen, daß auch dieser Fortschritt für sich allein höchstens zu einer etwas verbesserten Pflanzenkunde, niemals aber zu einer botanischen Wissenschaft geführt hätte. Kenntnisse bringen Wissen, aber noch keine Wissenschaft; diese setzt wissenschaftliche Betrachtungsweise, Denkmethode und Fragestellung 
voraus. Von all dem wußte das Mittelalter auf botanischem Gebiet nichts; erst das 16. Jahrhundert beschäftigte sich mit solchen Problemen und förderte sie. Gerade in dieser Beziehung war es entscheidend, daß damals die biologischen Werke Theophrasts bekannt wurden. Denn die Gelehrten des 16. Jahrhunderts mußten die biologische Betrachtungsweise, Denkmethode und Fragestellung nicht erst schaffen und aus dem Nichts aufbauen; sie fanden alles bereits in den Werken des großen Griechen. Es mag auf den ersten Blick merkwürdig berühren und wenig glaubhaft klingen, daß ein Werk, das zu jener Zeit schon etwa 1800 Jahre alt und an die 900 Jahre verschollen gewesen war, eine solche Bedeutung gehabt haben konnte. Es läßt sich aber nicht widerlegen, daß das erste Buch von Theophrasts «Historia plantarum», welche «de differentiis plantarum et partium» handelt, die erste, sehr bedeutsame morphologische Lehrfibel der angehenden Botaniker war und daß sich unter dem Einfluß dieses Werkes die morphologische Betrachtungsweise allgemein durchsetzte, welche die unbezweifelte Grundlage der wissenschaftlichen Botanik werden sollte. Erst jetzt begann man, nicht nur den Gesamthabitus einer Pflanze, sondern auch ihre einzelnen Teile näher anzusehen und unter einander zu vergleichen. Nur durch die konsequente morphologische Betrachtungsweise war es möglich, zunächst Genera und Species begrifflich genauer zu fassen, die schon im 16. Jahrhundert in höheren Einheiten zusammengefaßt wurden, wenn es auch noch nicht zu einer Aufstellung eigentlicher Familien kam. Hand in Hand damit gingen die ersten systematischen Bemühungen, die man bereits in verschiedenen Kräuterbüchern des 16. Jahrhunderts immer wieder feststellte, bei Lobelius, Tabernaemontanus u. a. ${ }^{27}$ Es ist ein Irrtum, die Botanik erst bei Caesalpin oder gar Jungius als wissenschaftlich anerkennen zu wollen. Ein System kann am Anfang einer neuen Entwicklungsepoche stehen; es wird aber in jedem Fall auch als Ende vorausgehender Arbeit zu verstehen sein, sozusagen als ihre Krönung. Ohne die eingehende floristische Tätigkeit des 16. Jahrhunderts, die zu einer ausgedehnten Kenntnis der einheimischen Pflanzenwelt geführt hatte, ohne die Aufstellung klarer Begriffe von Genera und Species, wie sie dieses Jahrhundert schuf, ohne die Ahnung von weitern, höhern Einheiten wäre weder das Bedürfnis nach einem umfassenden System entstanden, noch ein solches in Angriff genommen worden. Die Ehre, der erste wissenschaftliche Botaniker des Abendlandes genannt zu werden, wurde schon in früheren Jahrhunderten Conrad Geßner zugesprochen. Tournefort schrieb: 
Primus omnium qui veram aperuit viam ad plantarum cognitionem assequendam, generum adiumento, fuit Conradus Gesnerus ... Singulari enim nec alia usitata ratione icones stirpium depingi et sculpi fecit, plantas in partes dividendo et singula seorsim spectanda exhibendo. Mortuus est A. 1565, aetat. 49, cum fundamenta Botanices jaceret, quae pluribus deinde vitiis laboravit, quod eius vestigia non secuti fuerint qui post ipsum vixerunt. Fundamenta enim haec maxime ponebat in flore et fructu ... Primus autem qui post eum solida Botanices principia a fructificatione desumta constituit fuit Andreas Caesalpinus. ${ }^{28}$

A. v. Haller rühmte ihm in seiner Bibliotheca botanica nach:

Methodi botanicae rationem primus pervidit, dari nempe et genera quae plures species comprehenderent et classes quae multa genera. (Epist. p. 39 b) Varias etiam classes naturales in libro de collectione stirpium expressit. Characterem adfinitatum in flore inque semine posuit, in quibus depingendis ipse monet, se summo studio esse versatum. ${ }^{29}$

In der morphologischen Betrachtungsweise fußte Geßner auf Theophrast. Aber er blieb nicht nur dessen Schüler; sein Weg führte ihn über den Meister hinaus. Dieser hatte in Anlehnung an die Tierlehre von Aristoteles den bleibenden Pflanzenteilen, der Wurzel, dem Stamm und den Ästen, ausschlaggebende systembildende Bedeutung zugemessen, geringere den Blättern und die unwesentlichste den flüchtigsten Teilen, den Blüten und Früchten. Dem gegenüber erkannte bereits Geßner, daß gerade aus diesen die größere oder geringere Verwandtschaft der einzelnen Pflanzen am deutlichsten ersichtlich ist. Niemand kann im Ernst einer solchen Leistung das wissenschaftliche Prädikat absprechen. Von den Kräuterbüchern des 15. Jahrhunderts zu dieser Leistung ist es ein entscheidender Schritt, eben der Schritt von einer praktisch orientierten Pflanzenkunde zu einer wissenschaftlichen Botanik.

Aber nicht allein durch die morphologische Betrachtungsweise waren die Werke Theophrasts für diese Entwicklung bedeutungsvoll. In ihnen lernten die Gelehrten des 16. Jahrhunderts auch eine adäquate biologische Denkmethode und wissenschaftliche Fragestellung kennen, die damals völlig neu und dem ganzen Mittelalter unbekannt gewesen waren. G. Senn kam in jahrzehntelangen Theophrastforschungen zur Überzeugung, daß Theophrast die biologische Forschungsmethode bereits zu einer Vollkommenheit gebracht habe, daß sie in der Folge nicht mehr prinzipiell überboten werden konnte und noch heute ihre volle Geltung besitze, jene induktive Methode, die von sinnlichen Wahrnehmungen ausgeht, diese logisch miteinander verknüpft und daraus richtige Folgerungen ableitet, unter Heranziehung des Experiments, in welchem die 
Natur die an sie gestellten Fragen direkt beantwortet, sofern nur richtig gefragt wird. ${ }^{30}$ Die Beobachtungsmöglichkeiten haben sich seit seiner Zeit durch immer feinere Zergliederung und neue, vollkommenere Hilfsmittel ständig weiter entwickelt, wie sich auch die experimentellen Möglichkeiten qualitativ und quantitativ in einst ungeahnter Weise gesteigert und vermehrt haben - aber die allem zugrunde liegende Denkmethode wurde im Verlauf von jetzt mehr als zwei Jahrtausenden nicht grundsätzlich anders. Ihrer unwiderstehlichen Macht verdankte die Naturwissenschaft ihren Siegeslauf in erster Linie und dieser begann, als die theophrastischen Werke im Abendland wieder bekannt geworden waren. Biologische Naturwissenschaft gibt und gab es immer nur dort, wo diese Denk- und Forschungsmethode Geltung und Herrschaft hatten. Hier liegt die enorme Bedeutung von Theophrasts biologischem Werk. Dies verkannt und dasselbe nur auf seinen stofflichen Inhalt geprüft zu haben, war der Irrtum vieler älterer und neuerer Historiker der Naturwissenschaft. ${ }^{31}$ Mit Recht hat G. Senn in der Ausbildung dieser Methode die gültige Leistung Theophrasts erblickt. Die nicht weg zu leugnende Tatsache, daß man auch bei ihm zeitbedingte spekulative Eierschalen finden kann, die Feststellung, daß auch er in der polaren Spannung zwischen Spekulation und Empirie gestanden habe, ändern daran gar nichts. Auf seine entwicklungsträchtige, gültige Leistung allein kommt es an und diese hat G. Senn als erster klar erkannt.

Diese so lange vergessene Forschungsmethode findet sich in wenn auch noch bescheidenen Anfängen bereits bei der ersten Botanikergeneration des 16. Jahrhunderts, vor allem wieder bei Conrad Geßner. Es ist hier weder der Raum noch die Gelegenheit, dieser im einzelnen nachzugehen. Daß sie aber tatsächlich vorhanden war, mag folgendes Beispiel belegen. Als er einst ein Cichorium «caule fasciato» erhielt. dergleichen er selbst schon beobachtet hatte, schrieb er dem Sender: Tu futura aestate diligentius observabis: nam si ex huius semine alia nas. catur similia herba, rem secundum naturam esse coniicies, si non praeter naturam. ${ }^{32}$ Solche Versuche, solche direkte Fragen an die Natur, stellte Geßner in seinem botanischen Garten unzählige an. Nur dadurch kam er zu einer schärfern Erfassung der Species und auch zu einer Unterscheidung von Species und Varietäten. So bat er den Sender eines Blattes von Ilex aquifolia mit nur einem einzigen Stachel an der Spitze jedes Blattes, er möge doch untersuchen, ob dieser Unterschied konstant sei oder nicht. ${ }^{33}$ Einem andern Korrespondenten schrieb er: «Existimandum 
est autem nullas propemodo herbas esse quae non genus aliquod in duas aut plures species notae sunt. Gentianam unam prisci describunt, mihi decem aut plures species notae sunt»... ${ }^{34}$ Geßners Forschungsmethode war eine bereits wissenschaftlich zu nennende gewesen. Seine Lehrmeister, über die er freilich hinauswuchs, waren in erster Linie Theophrast, erst in zweiter Linie Dioskurid gewesen.

IV.

Die Werke Theophrasts fanden nördlich der Alpen schon früh Leser und Bewunderer. Diesem Studium entsprach eine vermehrte Nachfrage nach Theophrastdrucken. Von 1531-1541 war Basel mit seinen Theophrasteditionen führend; keine andere Stadt des Abendlandes konnte sich in dieser Beziehung mit seinen Leistungen vergleichen. Zumal in Deutschland war zu jener Zeit von einem Theophraststudium noch kaum zu reden. Die älteste Nachricht von einem schweizerischen Theophraststudium betrifft merkwürdigerweise einen Theologen, der sich naturwissenschaftlich in keiner Weise ausgezeichnet hat, nämlich Ulrich Zwingli. 1518 mußte ihm der Basler Buchhändler Amerbach eine lateinische Aldinaausgabe der Werke von Aristoteles und Theophrast beschaffen, die er beim Studium der griechischen Ausgabe benutzen wollte. ${ }^{35}$ Amerbach schickte ihm die Ausgabe von 1504, die sich, mit zahlreichen Randnotizen von $Z$ winglis Hand versehen, in der Zürcher Zentralbibliothek befinden soll. ${ }^{36}$

Auch der Zürcher Stadtarzt Dr. Christoph Clauser (gest. 1552) hat sich eingehend mit den Werken Theophrasts befaßt. Er besaß eine griechische Aldina und die lateinische Ausgabe «De historia et causis plantarum», die Wechel 1529 in Paris herausgegeben hatte. ${ }^{37}$ Die letztere findet sich als No. 26 aus Clausers Bibliothek, mit vielen Marginalien von Clausers Hand, ebenfalls im Besitz der Zentralbibliothek. Er scheint das ganze Werk von der ersten bis zur letzten Seite durchstudiert zu haben. Viele Fehler der lateinischen Übertragung Gaza's hat er an Hand des griechischen Exemplars verbessert. Bei allen ihm fraglich erscheinenden lateinischen Pflanzendiagnosen schrieb er den griechischen Pflanzennamen an den Rand. Er machte auch einen Auszug von allen Gewährsmännern, die Theophrast in seinem pflanzenkundlichen Werk aufführt. Bekanntlich war es vor allem Clauser, der seinen jüngern Kollegen Conrad Geßner zu ähnlichen Studien angeregt hat. ${ }^{38}$ Publiziert hat er freilich nichts was auf dieses Studium Bezug gehabt hätte. Im- 
merhin ersieht man aus seiner 1531 bei Froschauer in Zürich erschienenen Harnschrift, daß er neben den Werken Theophrasts auch das Kräuterbuch des Krataeus, die Werke von Dioskurid und Plinius gekannt hat, ${ }^{39}$ während er sich in späteren Jahren vor allem auch für Paulus Aegineta interessierte. ${ }^{40}$ Die eigentlichen Kräuterbücher, den Gart der gesuntheit und auch dasjenige von 0 . Brunfels scheint er eher abgelehnt zu haben. Sicher war Clausers pflanzenkundliches Interesse nicht ein wissenschaftlich-botanisches, sondern ausschließlich medizinisch-pharmazeutisches.

Otho Brunfels (gest. 1534 als Berner Stadtarzt) war zwar ein Mainzer von Herkunft; er hatte aber in Basel Medizin studiert und 1532 dort doktoriert und wahrscheinlich auch eine Baslerin geheiratet, war wie gesagt in Bern Stadtarzt gewesen und gehörte in seiner geistigen Formation dem oberrheinischen, baslerisch-elsässischen Kulturkreis an. ${ }^{41}$ Sein botanisches Hauptwerk, Herbarum vivae eicones, das von 1530 an bei Schott in Straßburg erschienen ist, dürfte zu einem guten Teil in der Schweiz entstanden sein. Es wird zwar vor allem wegen seiner trefflichen Pflanzen-Holzschnitte von Weiditz geschätzt, weniger wegen des ziemlich konventionellen Begleittextes von Brunfels. Man hat aber bis jetzt merkwürdiger Weise kaum je beachtet, daß es gleichzeitig ein erstes Sammelwerk wissenschaftlich-botanischer Literatur nördlich der Alpen darstellt. Brunfels selber steuerte eine erste Zusammenfassung der morphologischen Lehren Theophrasts aus dessen erstem Buch der Historia plantarum bei. ${ }^{42}$ Der zweite, 1531 erschienene Band enthielt ein Kapitel über Leonicens Pliniuskorrekturen (Nicolaus Leonicenus de falsa quarundam herbarum inscriptione a Plinio), sowie botanische Erstlingsschriften von Leonhard Fuchs und Hieronymus Bock, Tragus genannt, welch letztern Brunfels zur Herausgabe seines Kräuterbuches ermutigt haben soll.43 Fuchs steuerte eine Arbeit über die Pflanzennamen der alten Botaniker bei. Von Hermann von Neuenaar brachte er Studien mit der Kritik über die Nomenklatur Dioskurids, nach M. Möbius der ersten derartigen Arbeit aus Deutschland. ${ }^{44}$ Sicher wurde im Brunfelsischen Werk die morphologische Betrachtungsweise Theophrasts zum erstenmal im deutschen Sprachbereich ins helle Licht gerückt.

Der bedeutendste schweizerische Theophrastforscher war aber ohne Zweifel Conrad Geßner von Zürich (1516-1565). Im Lexicon Graecolatinum Basileense, das im Verlauf des 16. Jahrhunderts in verschiedenen Auflagen bei verschiedenen Verlegern erschienen war und an welchem 
Geßner von 1537 bis 1560 leitend mitgearbeitet hatte, ${ }^{45}$ fanden die Fortschritte der Theophrastinterpretation einen deutlichen Niederschlag. Nach Geßner übernahm Robert Constantin, ein Franzose, die Redaktion, der schon 1562 bei Crispin in Genf ein umfangreiches zweibändiges griechisch-lateinisches Wörterbuch herausgegeben hatte. ${ }^{46}$ Auch Constantin selber (gest. 1606) war ein bedeutender Theophrastforscher.

1542 publizierte Geßner seinen viersprachigen Catalogus plantarum. ${ }^{47}$ Er enthält die erste kritische Auseinandersetzung eines Schweizers mit Gaza's Theophrastinterpretation. Der junge Gelehrte zählte darin 125 Pflanzen auf, die ihm nicht diagnostizierbar erschienen.

1552 gab Geßner ein Buch De collectione stirpium ${ }^{48}$ heraus, das 1587 noch einmal in einer stark erweiterten von C. Wolf besorgten Auflage im Druck erschien. Es enthält als erstes in Deutschland erschienenes Buch eine ausführliche Lehre der Pflanzenmorphologie, in enger Anlehnung an das erste Buch von Theophrasts Historia plantarum, stellenweise auch an Dioskurid. In der Schilderung der Blüten und Früchte geht Geßner über Theophrast hinaus. Übrigens hatte er schon wenige Jahre vorher für den geplanten aber nie zur Ausführung gekommenen zweiten Band seiner Pandekten, der die Medizin und Naturwissenschaft enthalten sollte, Theophrasts Lehre De differentiis plantarum et partium in tabellarischer Form darzustellen versucht. Ein Fragment dieser Arbeit hat sich erhalten und wird heute in der Zürcher Zentralbibliothek im Mscr. C. 50 aufbewahrt, ohne bisher als solches erkannt zu werden.

Im Florilegium des Stobaeus ${ }^{49}$ hat Geßner auch Charakterbilder Theophrasts ediert. Stobaeus, angeblich ein Macedonier, stellte ums Jahr 500 für seinen Sohn eine Antologie aus den Werken von etwa fünfhundert griechischen Schriftstellern und Philosophen zusammen, Werken, die heute zum größten Teil verloren sind. Der Stoff ist thematisch angeordnet. Außer den Charakterbildern enthält das Werk offenbar auch Stellen aus einem heute verlorenen Werk Theophrasts über das Heiraten, das aber noch Diogenes Laertius gekannt hat, das Werk $\pi \varepsilon \varrho i ~ \gamma \alpha \dot{u} \mu \eta$. Geßner gab dieses Florilegium ein erstes Mal 1543 und nach einer bessern, aus Spanien stammenden Handschrift 1549 ein zweites Mal heraus. Philologisch sind diese Ausgaben nicht sehr geschätzt. Ihr Wert wäre vornehmlich in abweichenden Lesarten noch anderweitig vorhandener antiker Schriften zu erblicken gewesen. Geßner, der sich offenbar mehr stofflich für dasselbe interessiert hat, glich leider abweichende Lesarten den damals gebräuchlichen an. ${ }^{50}$ 
Nach Geßner hat sich in der Schweiz zunächst nur noch Caspar Bauhin von Basel (1560-1624) eingehender mit Theophrasts botanischen Werken befaßt, in Arbeiten, die allerdings bereits dem 17. Jahrhundert angehören, in dem 1601 erschienenen "Animadversiones in historiam generalem plantarum Lugduni editam» sowie vor allem in dem im Jahr 1623 im Druck erschienenen "Pinax theatri botanici», seinem bedeutenden nomenklatorischen Werk..$^{51}$

Über die Theophrastforschungen seines ältern Bruders Johann Bauhin (1541-1613), nicht nur des bedeutendsten Geßnerschülers, sondern nach Geßner wohl auch des bedeutendsten schweizerischen Botanikers des 16. Jahrhunderts, läßt sich nichts bestimmteres sagen. $\mathrm{Da} \beta$ sich, wie A. Burckhardt behauptete, die Animadversiones seines Bruders in erster Linie an seine Adresse gerichtet hätten, ${ }^{52}$ ist nicht anzunehmen; er wollte damit ohne Zweifel Jean Desmoulin (1530-1620) treffen, der die von Daléchamps und Johann Bauhin begonnene, aber erst später erschienene Lyoner «Historia plantarum generalis» nicht nur ediert, sondern nach zeitgenössischem Urteil ohne Zweifel auch verdorben hat. ${ }^{53}$

Gewiß gab es im 16. Jahrhundert in der Schweiz noch viele pflanzenkundlich interessierte Männer, die sich mit Theophraststudien abgaben, ohne daß wir davon Kenntnis haben. So wissen wir leider nicht, in wessen Besitz die prachtvolle griechische Aldinainkunabel der Zürcher Zentralbibliothek war. Als sie wohl im vorigen Jahrhundert neu gebunden werden mußte, mißfielen dem Buchbinder offenbar die handschriftlichen Randnotizen, sodaß er sie kurzerhand abschnitt, bis auf kümmerliche Reste, mit denen sich nichts mehr anfangen läßt. Einem großen Bedürfnis kam sicher die handliche, bei Wechel in Paris 1529 erschienene Theophrastausgabe entgegen, welche nur die Bücher «De historia et causis plantarum» enthielt mit griechisch-lateinischem und lateinischgriechischem alphabetischem Pflanzenverzeichnis, wie sie Clauser in Z̈̈rich besaß, wie sie sich aber auch in den Bibliotheken von Basel, Bern und St. Gallen findet, ohne daß wir ihre frühern Besitzer kennen würden. In Deutschland scheint diese Ausgabe eine Rarität zu sein, konnten sie doch die bedeutendsten deutschen Theophrastforscher bis auf Schreiber im beginnenden 19. Jahrhundert nie zu Gesicht bekommen. ${ }^{54}$ Die großen aldinischen Ausgaben, von denen Kapiteleinteilung und Pflanzenverzeichnisse übernommen worden waren, enthielten neben diesen Werken Theophrasts noch die aristotelischen Bücher De natura animalium, De partium animalium, De generatione animalium, sowie die Problemata 
von Aristoteles und von Alexander Aphrodisiensis. Diese dickleibigen Bücher waren natürlich entsprechend teuer. Abgesehen von der Zwinglischen Ausgabe wissen wir von keiner der in der Schweiz befindlichen, aus wessen Besitz sie stammt. Sicher ermöglichten erst die kleineren ein allgemeineres Theophraststudium, hinter dem das Studium der biologischen Werke von Aristoteles erheblich zurückgeblieben zu sein scheint.

V.

Die erste schweizerische Theophrastausgabe erschien bei Cratander in Basel, im Jahr 1531, ein reizendes Bändchen, das die fünfzehn damals bekannten Charakterbilder Theophrasts in griechischer Ursprache und lateinischer Übersetzung enthält. ${ }^{55}$ Wer diese Ausgabe besorgt hat, ist unbekannt. Sie stimmt weder im griechischen noch im lateinischen Text mit der vier Jahre zuvor in Nürnberg erschienenen, durch Willibald Pirckheimer besorgten Ausgabe überein, der im Jahr 1515 von seinem Freund G. Pico della Mirandola eine offenbar etwas flüchtige Abschrift erhalten hatte. ${ }^{56}$ C. Geßner bezeichnete zwar an verschiedenen Stellen den Florentiner Humanisten Angelus Politianus als den Autor der Basler Ausgabe. ${ }^{57}$ F. C. Mencken berichtet aber in seiner lateinisch verfaßten, 1736 in Leipzig erschienenen kenntnisreichen Biographie über den Florentiner, er habe im ganzen Schrifttum von und über Politianus nicht den mindesten Hinweis für die Richtigkeit dieser Angabe finden können, sodaß er die Frage dieser Autorschaft offen ließ. ${ }^{58}$ Offen ist sie auch heute noch, da keine neuen Momente hinzugekommen sind, die eine Beantwortung erlauben würden.

1534 erschien im selben Verlag ein Neudruck der aldinischen lateinischen Ausgabe von 1513, mit geringfügigen textlichen Verbesserungen, ohne die Problemata des Alexander Aphrodisiensis, mit gleicher Kapiteleinteilung und gleichen Verzeichnissen. ${ }^{59}$ Die umfangreiche und darum wohl wenig begehrte Ausgabe fand offenbar nicht den gewünschten Absatz, sodaß sie heute auch in der Schweiz selten anzutreffen ist. Das Berner Exemplar trägt den Besitzervermerk von zwei Basler Buchdruckern, Walders und Laurentius Hospinians. Daß die Wechelsche kleinformatige Ausgabe trotz vermehrten Fehlern einem Bedürfnis entsprach, ersieht man daraus, daß der Verlag von Cratanders Erben 1550 eine gleiche Ausgabe auf den Markt brachte ${ }^{60}$ und kurz darauf auch Rovillius in Lyon, von Jordanus allerdings neubearbeitet und mit einem Vorwort versehen. Die Lyoner Ausgabe enthielt auch die Praefatio von 
Gaza sowie dessen Brief an Papst Nicolaus V., in dem er seine Schwierigkeiten in der Theophrastinterpretation schildert. Das Basler Exemplar dieser Ausgabe gehörte erst einem Oswald Schreckenfuchs, später Caspar Bauhin. ${ }^{61}$

Wichtiger als alle diese Ausgaben war die griechische Edition aller damals bekannten Theophrastwerke durch Oporin vom Jahr $1541 .{ }^{62}$ Es handelte sich um eine handliche Kleinfolio-Ausgabe von mäßiger Dicke. Dieser Vorteil konnte allerdings nur durch einen recht engen Satz erkauft werden, der die Lesbarkeit nicht eben erleichtert. Außer den bereits erwähnten Pflanzenbüchern enthält die Ausgabe verschiedene kleinere Arbeiten Theophrasts, die teilweise hier zum erstenmal zum Druck kamen, wie auch Theophrasts Lebensgeschichte von Diogenes Laertius mit dessen Verzeichnis von sämtlichen ihm bekannt gewesenen Theophrastwerken, die heute zum großen Teil verloren sind. Diese Biographie, die allerdings reichlich fünfhundert Jahre nach Theophrasts Tod erschienen ist, bildet neben einzelnen Aussprüchen Plutarchs und Ciceros unsere einzige Nachrichtenquelle über das Leben des griechischen Vaters der Botanik. Bei den kleinern Schriften handelt es sich um jene über die Steine, die Fische, das Feuer, die Winde, die Zeichen des Wassers und der Winde, den Augenschwindel, die Arbeit, die Düfte, die Schweiße und die damals bekannten fünfzehn Charakterbilder, deren Zahl sich später auf dreißig erhöhte. In der Version Priscians enthält die Ausgabe die angeblichen Theophrastwerke über die Phantasie und das Gefühl. Oporin lieferte nicht nur die erste Gesamtausgabe der Werke Theophrasts, sondern gleichzeitig die erste kritische Edition seit der Ausgabe von Gaza, welche die textkritische Arbeit des ersten halben Jahrhunderts seit Erscheinen dieser Werke nach Möglichkeit verwertete. Auch dieser Ausgabe war der Text von Gaza zu Grunde gelegt, wo ein solcher vorhanden war. In Randnotizen wurden viele verbesserte Lesarten angegeben. Die meisten haben späterer Kritik standgehalten und bilden heute einen Bestandteil des Textes. Merkwürdiger Weise gibt es zwei verschiedene Fassungen derselben Ausgabe; die eine ist mit einem Vorwort des aus dem Elsaß stammenden, damals an der Basler Hochschule als Altphilolog wirkenden Hieronymus Gemuseus versehen ${ }^{63}$ die andere mit einem Vorwort des später vor allem in Leipzig wirkenden berühmten Philologen Joachim Camerarius. ${ }^{64}$ Hoffmann, der bedeutende deutsche Theophrastforscher und Medizinprofessor in Altdorf bei Nürnberg meinte, wie man in der Praefatio der verdienstvollen Theophrastausgabe Schnei- 
ders (1818 in Leipzig erschienen) nachlesen kann, etwas boshaft, das Werk habe sich offenbar schwer verkaufen lassen, weshalb das Vorblatt mit der Einführung von Gemuseus herausgerissen worden und durch eine solche des berühmteren Camerarius ersetzt worden sei. ${ }^{65}$ Das genaue Gegenteil dürfte zutreffen. Offenbar fand die Ausgabe so guten Absatz, daß bald, noch im selben Jahr, ein Neudruck nötig wurde. Da die erste Fassung anonym erschienen war, legte Oporin begreiflicher Weise Wert darauf, sich in der zweiten als Autor der Textkritik vorzustellen, was durch Camerarius in höchst anerkennender Weise geschah. Dieser Name allein ist tatsächlich eine Bürgschaft. Bei der Abfassung der Marginalien war Oporin so vorgegangen, daß er sich von verschiedensten Gelehrten ihre Handexemplare ausgebeten hatte, ihre Randnotizen miteinander vergleichend und gegen den Text von Gaza abwägend. Dazu war Oporin besonders befähigt, war er doch nicht nur naturwissenschaftlich gebildeter Mediziner, sondern gleichzeitig ein vorzüglicher Altphilologe, der während mancher Jahre die Griechischprofessur der Basler Hochschule bekleidet hatte. ${ }^{66} \mathrm{Da}$ ihm neue Handschriften nicht zur Verfügung standen, hatte er kaum eine andere Möglichkeit, den stellenweise sehr verderbten und geradezu unverständlichen Text in Gaza's Fassung zu verbessern. Seine Marginalien stellen ohne Zweifel eine Kollektivleistung verschiedenster Gelehrter dar und sind der Niederschlag einer ersten Theophrastforschung. Camerarius spricht denn auch von einer «editio a multis mendis doctorum virorum industria et exemplorum meliorum ope purgata». Vom botanischen Hauptwerk Theophrasts existieren ja im Grunde genommen auch heute nur zwei in der Fassung verschiedene Handschriften, von denen die andern irgendwie abstammen, die mediceische und die weitaus bessere urbinatische, die sich in der Vaticana findet. ${ }^{67}$ So muß Oporins Vorgehen ja bekanntlich auch heute noch in der Theophrastforschung und Interpretation angewandt werden. Diese griechische Theophrastausgabe gehörte zu den Großtaten der Oporin'schen Offizin; sie wurde in der Folge die Mutter aller folgenden kritischen Theophrasteditionen. Auf welche Weise sie zu solchen benutzt wurde, zeigt deutlich das Berner Exemplar mit seinen zahlreichen Randnotizen von verschiedenster Hand, dessen Herkunft leider in Dunkel gehüllt ist. ${ }^{68}$

Wenn man bedenkt, daß Oporin kaum zwei Jahre später die Prachtausgabe von Vesals Fabrica humana herausbrachte, kann man ermessen, zu welchem Dank Naturwissenschaft und Medizin diesem trefflichen 
Mann verpflichtet sind, den eine gewisse Paracelsusliteratur ganz zu Unrecht zu einer lächerlichen Figur zu machen versucht hat wegen seiner abschätzigen Bemerkungen über Paracelsus, dessen Famulus er eine Zeitlang gewesen ist, Bemerkungen, die offenbar nicht für den Druck bestimmt waren. In seinem Leben hat Oporin viel Unglück gehabt, als Famulus von Paracelsus, als Griechischprofessor an der Basler Hochschule, da er diese Stelle nach dem Zeugnis Conrad Geßners durch iniuria verlor, ${ }^{69}$ in seinen häuslichen Verhältnissen, mit seinen Geschäftspartnern, die ihn, wie Robert Winter, betrogen, in seinen Geschäften überhaupt, um deren kaufmännischen Teil er sich zu wenig bekümmert hatte, sodaß er in Armut, Elend und Not gestorben ist. Aber eine lächerliche Gestalt war er deshalb ganz bestimmt nicht. Noch heute zeugen seine Werke für ihn ${ }^{70}$, und Geßner nannte ihn in seiner Bibliothek «omnium typographorum nostra aetate doctissimus».

VI.

Zum Schluß noch einige Bemerkungen über Theophrastmanuskripte in schweizerischen Bibliotheken. Es sind ihrer naturgemäß nicht viele. Das schönste besitzt die Berner Stadtbibliothek in Mscr. 402, einem schönen, griechisch geschriebenen Sammelband, der außer kleinern Schriften Theophrasts auch Werke von Aristoteles enthält, wie seine «Mechanica» mit interessanten Zeichnungen. Von Theophrast finden sich die Schriften über das Feuer, die Steine, die Schweiße, den Schwindel, die Arbeit, die Fische, die Winde und die Düfte. Die Handschrift ist zwischen 1480 und 1490 in Italien entstanden und älter als alle Drucke, wenigstens, was die Theophrastschriften betrifft. Der schöne Ledereinband soll venetianischer oder florentiner Herkunft sein. Das Vorsatzblatt mit dem Wasserzeichen Briquet 2497 weist nach Venedig (1480-84); das für den Text benutzte Papier mit dem Wasserzeichen Briquet 92 soll 1479 in Udine hergestellt worden sein, Mitteilungen, die ich der Freundlichkeit von Herrn Dr. Strahm von der Berner Stadtbibliothek verdanke.

Die Stadtbibliotheken von Bern und St. Gallen besitzen zwei ziemlich gleichlautende lateinisch verfaßte Handschriften aus dem 15., eventuell 16. Jahrhundert, welche im Titel Theophrast zugeschrieben sind, in der Theophrastliteratur aber nirgends erwähnt werden. Mscr. 531 der Berner Stadtbibliothek enthält in No. 12 auf drei Seiten den «Tractatus de Theophrasti libro de nuptiis. Fertur aureolus theophrasti liber de nuptiis in quo querit an vir sapiens ducat uxorem etc.». Mscr. 483 der 
St. Galler Vadiana zeigt in No. 3 ebenfalls einen Tractatus Theophrasti de nuptiis als «Aureolus Theophrasti liber in quo querit, an vir sapiens ducat uxorem», gleichfalls auf drei Seiten. Die St. Galler Handschrift wurde, worauf zuerst Dr. M. Gabathuler von der Vadiana aufmerksam gemacht hat, von G. Scherrer, dem Verfasser des St. Galler Handschriftenkata$\operatorname{logs}$, Paracelsus zugeschrieben, wohl weil er irrtümlich Aureolus auf Theophrast statt auf liber bezog. Der Inhalt dieser Schrift, der die Ehe für einen Philosophen für abträglich hält, wurde auch in Albrecht von Eybs 1472 bei Günther Zainer in Augsburg erschienenes Ehebüchlein aufgenommen. "Ob einem mann sei zu nemen ein eelich weib oder nit», von welchem die St. Galler Stadtbibliothek ein Exemplar besitzt. Albrecht war deutscher Frühhumanist und Domherr. ${ }^{71}$ Die Berner wie die St. Galler Handschrift gehörten offensichtlich Klerikern. Ein griechisches Vorbild zu dieser Arbeit ist unbekannt, wenn sie auch inhaltlich mit einschlägigen Theophraststellen im Florilegium des Stobaeus merkwürdig

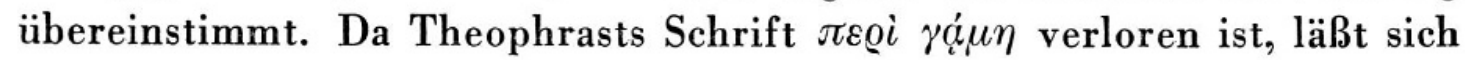
nicht bestimmen, ob diese als Vorbild gedient hat oder ob es sich lediglich um ein Klerikerprodukt des 15. Jahrhunderts handelt.

Bernhard Milt.

\section{Literaturnachweise}

1. Diogenes v. Laerte aus Cilicien, etwa 220 n. Chr., schrieb zehn Bücher über «Leben, Ansichten und Aussprüche berühmter Philosophen». Das 5. Buch handelt von Theophrast und bringt ein ausführliches Verzeichnis seiner heute weitgehend verlorenen Werke. Oporin nahm diese Biographie in seine 1541 erschienene griechische Gesamtausgabe der Theophrast'schen Schriften auf. Das Werk von Diogenes Laertes wurde 1828 ff. von Hübner, Leipzig, neu herausgegeben. - 2. H. Fischer, Mittelalterliche Pflanzenkunde, Münchner Drucke, 1929./ M. Möbius, Gesch. d. Botanik, G. Fischer, Jena, 1937, S. 13 ff. - 3. Theophrasti Eresi quae supersunt opera, hgg. v. Schneider, Leipzig, 1818, 1. Bd., Praefatio (ex excerptis C. Hoffmanni hist. nat. Theophrasti pertinent.). - 4. B. Milt, Beitr. z. mittelalt. Heilk. a. Bodensee u. Oberrhein, Viertelj'schr. d. Natf. Ges. i. Zürich, 85. Jgg., H. 3-4, S. 282. - 5. H. Fischer, 1.c., S. 125 / Cd.Geßners bot. Nachlaß, photogr. u. photokop., Z. B. Zürich.6. H. Fischer, 1. c., Pflanzenbilder d. Mittelalters, S. 114 ff. - 7. G. Toffanin, Humanismus, Akad. Verlag Pantheon, 1941./ G. Voigt, Die Wiederbelebung d. klass. Altertums, Berlin, 1859, bes. 5. Buch./ Patrologiae cursus completus, hgg. v. Migne, CLXI, Leben und Werke Bessarions; sein Brief an Th. Gaza über seine Interpretationen, S. 686. - 8. Schneider, 1. c., Praefatio. / Brief Th. Gaza's an Papst Nicolaus V., abgedr. i. verschiedenen Theophrasteditionen, z. B. der Lyoner Ausgabe De Historia et causis plantarum Theophrasti, 1552. - 9. Ibidem. / Nicolai Leoniceni vincentini de Plinii et plurium aliorum medicorum in medicina erroribus op. I. Angelo 
Politiano dedicatum v. 3. I. 1491, gedr. i. Ferrara, 1509, S. 23 b: «Erat autem (nisi fallor) ea tua cogitatio quod Plinius non recte imitatus Theophrastum librum»./ Leonicens 4. Brief i. diesem Werk, S. 94a: «Errores Plinii ex similitudine graeci vocabuli» / Brief v. Th. Gaza an Papst Nicolaus V., 1. c. - 10. Schneider, l. c. / i. Zürich

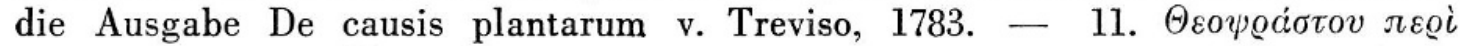


12. Biogr. Lex. d. hervorr. Ärzte aller Zeiten u. Völker, 2. Aufl., Berlin-Wien, 1929, Bd. 1, S. 322. - 13. Ibidem, Bd. 3, S. 744. - 14. Ibidem, Bd. 1, S. 454. - 15. Leonicen, l. c., S. 23 a. - 16. O. Vasella, Unters. ü. d. Bildungsverh. i. Bist. Chur usw., Chur, 1932 i. 62. J'Bericht d. hist.-antiq. Ges. i. Chur. - 17. A. Braun, D. Klerus i. Bist. Konstanz i. Ausgg. d. Mittelalters, Münster, 1938. - 18. P. Staerkle, Beitr. z. spätmittelalt. Bildungsgesch. St. Gallens, St. Gallen 1939, i. St. Galler Mitt. z. vaterl. Gesch., Bd. XL. - 19. Ibidem, Tab. I, S.110. - 20. G. Toffanin, 1. c., S. 377 ff. / H. Rüdiger, Wesen und Wandlung d. Humanismus, Hamburg, 1937, S, 103 ff. / Walter Rüegg, Cicero und der Humanismus, Zürich, 1946, S. 117 ff., freilich etwas anders gesehen. / Die reichlich gegensätzlichen Darstellungen beweisen vor allem, daß das Erasmusproblem noch in keiner Weise gelöst ist. Auch die Erasmusdarstellung von Huizinga kann in dieser Beziehung nicht befriedigen. Das Problem Erasmus-Reformation überschattet in den meisten Darstellungen das nicht minder wichtige Problem Erasmus-Rom und römischer Humanismus. Die Beziehungen von Erasmus zu den römischen Päpsten ist hinlänglich dargestellt in Th. Quoniam, Erasme, Paris, 1935, diejenigen zum römisch-lateinischen Humanismus sind bei Rüegg unter dem alleinigen Gesichtswinkel Ciceros zu eng gefaßt und dies um so mehr, als der Verfasser fast nur den formalbildenden Gesichtspunkt beachtet. - 21. H. Rüdiger, 1. c. S. 93, / Rupprich, Celtis-Briefwechsel, München, 1934, / G. Bauch, Anfänge des Humanismus i. Ingolstadt, München, 1903, / derselbe, Rezeption des Humanismus i. Wien, Dresden, 1903. - 22. W. Näf, Vadian, St. Gallen, 1944, S. $263 \mathrm{ff}$. - 23. Vadiani loca aliquot ex Pompinianis commentariis repetita, Basel, 1522, unpag. - 24. R. Burckhardt, Gesch. d. Zoologie, Samml. Göschen No. 357, Leipzig, 1921, S. 57 f.; da B. in Geßner nur einen Kompilator sieht, der «die Kompilation zur selbständigen Kunst entwickelte», rechnet er Geßner unter die philologischen Naturwissenschafter, auch wenn er ihn ein Kapitel später bespricht. - 25. C. Gesneri catalogus plantarum, Zürich, 1542, S. 159 b. - 26. H. Fischer, l. c., S. 122. - 27. M. Möbius, 1. c., S. 28, 32. - 28. Zitiert nach J. Geßners Diss. de vegetabilibus, Zürich, 1740, S. 19; Tournefortii institut. rei herbariae, Paris, 1700, standen mir nicht zur Verfügung. - 29. A. Haller, Bibl. botanica, Zürich, 1777, S. 284. - 30. G. Senn, Die Entwicklg. d. biolog. Forschungsmethode i. d. Antike u. ihre grundsätzliche Förderung durch Theophrast v. Eresos, Aarau-Leipzig, 1933, Veröffentl. d. schw. Ges. d. Medizin u. Naturw., Bd. 8. - 31. J. Sachs, Gesch. d. Botanik, München, 1875, S. 17, / R. Strömberg, Theophrastea, Göteborg, 1937 i. Göteborgs kungl. Vetenkaps-och Vitterhets-Samhälles Handlingar, S. 179. 33. C. Gesneri epist. medic. libri III, Zürich, 1577, S. 94 a. - 34. Ibidem, S. 86 a. 35. W. Köhler, Zwinglis Bibliothek, i. Zwingliana, Bd. 4, S. 60, / im Amerbach-Briefwechsel (hgg. v. A. Hartmann, Basel, 1942 ff.) findet sich die Mitt. Amerbachs, daß er Zwingli 1518 eine betr. Ausgabe besorgt hat. - 37. Die griech. Aldina 
Clausers läßt sich heute nicht mehr nachweisen; die latein. Wechelsche Ausgabe de hist. et causis plantarum, Paris, 1529, No. 26 von Clausers Bücherei, trägt die Signatur d. Z. B. Z.: RR 1621, / Über Clauser vgl. G. A. Wehrli, Dr. Christoph Clauser, Zürich, 1925, Bd. 2 d. Veröffentl. d. schw. Ges. f. Gesch. d. Medizin u. Naturw. - 38. C. Gesneri historia plantarum, Basel, Paris, Venedig, 1541 i. einleitenden Brief an H. Billing: «Cum superioribus mensibus suavissimam patriam Tigurum invisissem, salutandi gratia semel atque iterum doctissimum virum Christopherum Clauserum urbis nostrae archiatrum accessi. Is cum varia collequentibus nobis, medicinae studiosis rem utilem facturam diceret, si quis simplicium medicaminum e Dioscoride quaedam descriptiones, facultates autem ex Aegineta conscriberet, et iam prius saepe in mentem venerat.» - 39. G. A. Wehrli, 1. c., S. 77. - 40. Geßner an Billing, 1. c. - 41. F. W. E. Roth, Otto Brunfels, i. Jahrb. f. Gesch., Sprache u. Lit. Elsaß-Lothringens, Bd. XVI, 1900, / Möbius, 1. c., S. 26 f. - 42. Herbarum vivae icones, per Otho Brunfelsium recens editae, MDXXX, Argentorat.; Contenta appendicis sequentis: De differentiis herbarum historiae plantarum Theophrasti; De differentiis herbarum variis; Differentiae seminum, succorum, odorum, saporum, arborum, florum usw.; Differentiae radicum. - 43 und 44. Novi herbarii tom. II per Oth. Brunfelsium recens editus MDXXXI, Argentorat.; De vera cognitione herbarum appendix cui sequentia insunt: 6. Leon. Fuchsii, principis Brandeburg. medici annotat. de simplicibus, a medicis hactenus perperam intellectis et aestimatis. 7. Generosi Comitis Heremanni a Nevenar censurae aliae herbarum super eadem re.l Möbius, l. c., S. 21 u. 27. - 45. C. Gesneri ... de libris a se editis epistola usw. Zürich, 1562, No. 1: Lexicon Graecolatinum. - 46. Larousse, Grand dictionnaire universel du XIX. siècle, tom. 4, S. 1020. - 47. C. Gesneri Catalogus plantarum Latine, Graece, Germanice et Gallice, Tiguri, MDXLII. His accedunt i. Calce op. Nomenclaturae stirpium sec. varias gentes, Dioscoridi adscriptae, in ord. lit. digestae. - 48. C. Gesneri de Collectione stirpium tabulae, hgg. von C. Wolf, Zürich, 1587 / Tabulae collectionum quibus per singulos anni menses quae stirpes in singulis per Germaniam flores, fructusque proferant, ordine recensetur. Argent., MDLIII / Fragment einer tabellarischen Übersicht de differentiis plantarum et partium an Hand von Theophrasts 1. Buch der Hist. plantarum, von Geßner offenbar für den nie erschienenen 2. Bd. d. Pandekten gemacht, findet sich i. d.Z.B.Z. i. Mscr. C. 50 a, No. X. - 49. Joh. Stobaeus, ein griech. Schriftsteller wahrsch. aus Stobi i. Macedonien, lebte i. 5. od. 6. Jhd. n. Chr. Sein Gesamtwerk wurde neu herausgegeben 1884-1894, v. Wachsmuth u. Hense, Berlin. - 50. Über die Ausgaben vergl. Hoffmanni Lexicon bibliographicum, Lips. 1836, Bd.3, S. 631-633. 51. C. Bauhini Pinax theatri botanici, Basel, 1623, vgl. Möbius, 1. c., S. 38. 52. A. Burckhardt, Gesch. d. mediz. Fakultät z. Basel, Basel, 1917, S. 122. 53. Schneider, 1. c. / Larousse, 1. c., Bd. 6, S. 570. - 54. Schneider, 1. c. - 55. Theophrasti Characteres, Graecelatine, Basilea, 1531, ein reizendes Büchlein. - 56. Bilibaldi Pirckheimeri opera, edit. a Melchiore Goldasto, Francof. 1610, S. 15 u. 23 / Charakteren S. 214-228; Pico's Brief an Pirckheimer S. 212 / Über Pico's Beziehungen z. Pirckheimer vgl. H. Rupprich, Willibald Pirckheimer u. d. erste Reise Dürers n. Italien, Wien, 1930, S. 18. - 57. C. Gesneri Bibliotheca universalis pg. 614. - 58. Politianusausgaben d. 15. Jhds. m. Inhaltsverzeichnis i. Catalogue of 
books in the XVth century, now in the British museum, Abtlg. Venice, S. 559; es findet sich keine einzige von Politianus edierte oder kommentierte Theophrastschrift aufgeführt; / F. O. Mencken, Hist. vitae et in lit. merit. Angeli Politiani, Lips., 1736, S. 163. - 59. Findet sich i. d. Schweiz i. d. Univ.-Bibl. v. Basel u. Bern. - 60. Univ.-Bibl. Basel. - 61. Oswald Schreckenfuß, Dr. med. v. Basel, 1571; Stadtarzt v. Mülhausen, 1575; vgl. A. Burckhardt, l. c., S. 127. - 62. S. F. G. Hoffmann, Lexicon bibliographicum sive index editionum scriptorum Graecorum, Bd. 3, Lips., 1836, / Schneider, Praefatio, 1. c. - 63. A. Burckhardt, 1. c., S. 42 f. m. Lit. Angaben / C. Gesneri Bibliotheca universalis, bes. die Ausgabe von 1583, S. 348. - 64. J. Camerarius (1500-74) galt an kritischer Schärfe als der tüchtigste Altphilologe seiner Zeit. - 65. Vero cum non ex voto inveniret emtores, abiecta philyra prima praescripsit nomen Camerarii qui etiam praeloquium aliud fecit, in quo diserte affirmat, marginalia illa esse Oporini. - 66. C. Gesneri Bibliotheca universalis, Editio 1545 S. 446, bes. ausführlich editio 1583 (J. Fries), S. 480, / R. Wolf, Biogr. z. Kulturgesch. d. Schweiz, Bd. 3, S. 45-46/ A. Burckhardt, 1. c., S. 31. 67. O. Kirchner, Diss. «De Theophrasti libris phytologicis», Breslau, 1874; i. Zürich findet sich ein Exemplar i. d. Handbibliothek d. Instituts f. spez. Botanik d. ETH. - 68. Die i. Catalog. codic. Bernens. angegebene Signatur 0106 fol. lautet heute N 127. - 69. C. Gesneri Bibliotheca universalis, Editio 1583. - 70. Ibidem. 71. Nadler, Lit. Gesch. d. deutschen Volkes, Propyläenverlag, Berlin, Bd. 1, 1939, S. 288.

\section{Buchbespredhungen}

R. Strömberg: Griechische Pflanzennamen. Göteborgs Högskolas Årsskrift, Bd. 46 1940 ; 161 Seiten Text, Literatur- und Sachverzeichnis, 19 Seiten Pflanzennamenregister.

Der Verfasser dieser Arbeit gehört zu den bedeutendsten Theophrastforschern der Gegenwart; als solcher hat er sich durch seine 1937 erschienenen "Theophrastea, Studien zur botanischen Begriffsbildung» ausgewiesen. Im Vorwort dieses neuen Werkes sagt er: «Das Schwergewicht dieser Arbeit ruht in dem Bemühen, die griechischen Pflanzen nach ihren Benennungsgründen anzuordnen und an Hand von sicher zu bestimmenden Beispielen einen festen Grund für die Etymologie und Morphologie ihrer Namen zu legen.»Die Identifizierung griechischer Pflanzennamen steht alo nicht im Vordergrund seines Interesses und wird nur herangezogen, sofern sie zur Aufhellung namenbildender Elemente führt. Strömbergs Verdienst ist um so größer, als er sich diese Aufgabe für die griechischen Pflanzennamen als erster gestellt hat. Natürlich ist eine solche Arbeit nicht frei von hypothetischen Annahmen. Möglicherweise wird in Zukunft das eine und andere Urteil modifiziert werden; das würde aber an der grundlegenden Bedeutung der Arbeit kaum etwas ändern. Diese selber gliedert sich in 